\title{
Free fall water entry of a wedge tank into calm water in three
}

\section{degrees of freedom}

\author{
Shiyan Sun ${ }^{\mathrm{a}}$, Guoxiong $\mathrm{Wu}^{\mathrm{b}, \mathrm{a}, *}$, Gang $\mathrm{Xu}^{\mathrm{a}}$ \\ ${ }^{\text {a }}$ School of Naval Architecture \& Ocean Engineering, Jiangsu University of Science and \\ Technology, Zhenjiang 212003, China \\ ${ }^{\mathrm{b}}$ Permanent address: Department of Mechanical Engineering, University College London, \\ Torrington Place, London WC1E 7JE, UK
}

\begin{abstract}
The hydrodynamic problem of a two dimensional wedge tank filled with liquid entering a calm water surface is analysed based on the incompressible velocity potential theory. The motion effect of inner liquid on the entry process is investigated through comparison with the result containing equivalent solid mass or the liquid being frozen. The problem is solved through the boundary element method in the time domain. Two separated computational regions are constructed. One is the inner domain for the internal liquid, and the other is the outer open domain for the open water. The former is solved in the physical coordinate system, and the latter is solved in a stretched coordinate system. The solutions of two separated domains are connected through the motion of the body. The auxiliary function method is extended to decouple the nonlinear mutual dependence between fluid loads from two separated domains and the body motion. Detailed results for wedge motion, external impact pressure and free surface, and for internal pressure, free surface deformation and liquid motion are provided. Through comparison with the results of a wedge tank with frozen ice, in-depth discussion on the effect of the inner liquid is provided.
\end{abstract}

Key words: Water entry of a wedge tank; Fully nonlinear boundary conditions; Boundary element method; Dual domain problem; Free fall motion in three degrees of freedom.

\section{Introduction}

Fluid/structure impact is always a major concern in naval architecture and ocean engineering. It usually occurs when the structure penetrates liquid surface at a large relative speed. Examples include green water on deck, ship slamming, and overturning and breaking wave impact on offshore and coastal structures. Severe danger can be posed to marine structures by violent waves from the sea, and damage or even destruction can be caused. The impact usually lasts for a very short period of time, during which the physical parameters such as pressure and fluid particle acceleration are very high and change rapidly. The process is highly complex. This is further completed when a ship contains a liquid cargo, such as a LNG, or a ship is loaded with ballast.

One of the methods for water entry is based on the Wagner theory together with some asymptotic analysis. The wave elevation is obtained through the integration of fluid particle velocity on the free surface with respect to time, the wetted body surface is altered accordingly. The velocity potential on this previously dry surface of the body, which was not accounted for initially, should then be included. This means that there will be some implicit coupling between the potential and the area of the wetted body surface. Based on this procedure, Howison et al. [1] gave an explicit solution for a body with small deadrise angle. Korobkin et al. [2] adopted a numerical scheme for a body with

* Corresponding author.

Permanent address: Department of Mechanical Engineering, University College London, Torrington Place, London WCIE 7JE, UK. Tel.: +44 207679 3870; fax: +44 2073880180.

E-mail address: g.wu@ucl.ac.uk (G.X.Wu) 
elastic deformation. Later Korobkin [3] investigated the wave impact with the second order Wagner theory. The water entry problem has also been solved based on the fully nonlinear velocity potential theory in which the nonlinear boundary conditions are imposed on the instantaneous positions of the body surface and the free surface. The typical work includes those by Dobrovol'skaya [4] and Zhao \& Faltinsen [5] for the vertical entry of a symmetric wedge, Semenov \& Iafarati [6] for an asymmetric wedge, $\mathrm{Xu}$ et al.[7] for the oblique entry of an asymmetric wedge, and by $\mathrm{Wu}$ [8] for the problem of twin wedges. While all these are based on the constant entry speed, Wu et al. [9] considered vertical entry of a wedge in free fall motion with a single degree of freedom, and $\mathrm{Xu}$ et al. [10] considered the free fall with three degrees of freedom, in which the vertical motion is coupled with the horizontal and rotational motions. More recently, the body entering into a wave field instead of calm water was considered by Sun et al. [11, 12], and the flow detachment by Bao et al. [13, 14] for a finite wedge and cavity effect by Sun et al. [15] for a semi-circle. Other relevant work includes those by Wang and Faltinsen [16, 17] and Barjasteh et al. [18].

The present work considers the problem of a solid tank filled with liquid entering the water surface, which differs from those problems considered above. It may seem that the liquids inside and outside the tank are completely separated and their motions are therefore decoupled. However, when the tank is in the free motion, its motions will be affected by both internal and external fluid flows, which will in turn be affected by the body motion. Therefore all these three are fully coupled. It is well known that the liquid inside a tank may experience sloshing motion, which itself is a highly complex problem [19] and there have been extensive studies on this subject [20-24]. The sloshing motion is significantly affected by its natural modes. During water entry, however, the time scale of interest is usually much smaller than the natural periods of the tank. Therefore it is normally unlikely that it will be affected by the resonance modes of the internal fluid. It then may appear that within such a short time duration, the liquid motion is small and it can be treated stationary, or treated even as a solid mass like frozen ice. However, it can be shown that the motion characters of a tank with a liquid and with a solid mass are different. This is the main motivation of the present work.

As flow crosses the tip of wedge, a vortex sheet will be generated and be shed from the tip of the wedge. However, its effect is usually very localized and it may not significantly affect the overall hydrodynamic force and fluid flow. It is shown in Barjasteh et al. [25] that the numerical results without vortex shedding by $\mathrm{Xu}$ et al. [7] are in good agreement with the experimental data for the water entry of asymmetric wedges. Semenov and $\mathrm{Wu}$ [26] did include the vortex sheet from the tip of a wedge in their mathematical model. It was found that the effect of vortex was confined in a small local region, and the overall pressure distribution in other places is not significantly affected and the effect on the free surface is hardly visible. The analysis in this paper is based on the incompressible velocity potential flow theory without vortex sheet. The boundary element method (BEM) is used at each time step to solve the governing Laplace equation. Two separated computational domains are constructed, one is the inner domain, and the other is the outer open domain. The former is solved in the physical coordinate system, and the latter is solved in a stretched coordinate system [9]. The solutions of two separated domains are connected through the motion of the body. The auxiliary function method [27] is extended to decouple the nonlinear mutual dependence between fluid loads from two separated domains and the body motion. The effect of the inner liquid of the wedge tank on its water entry of is investigated. A typical feature is that the liquid seems be 'lighter' than the equivalent solid mass, in the sense that wedge will be more responsive to the external impact load. In other words, the acceleration of the tank with liquid is usually larger 
and more oscillatory. Extensive simulations are undertaken for different depths and densities of internal liquid, as well as the initial velocity of the wedge tank. The results are analysed and the implications in physics are discussed.

\section{Mathematical model and numerical procedure}

\subsection{The physical parameters and Cartesian coordinate system}

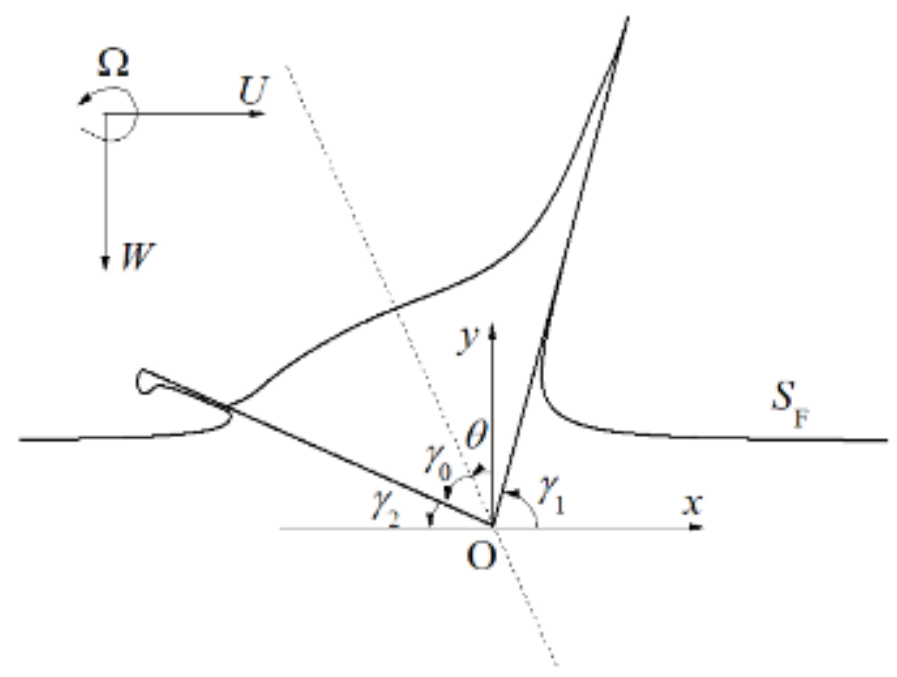

Fig. 1. The sketch of problem.

The sketch of the 2-D problem considered here is given in Fig. 1 which shows a wedge shaped tank entering into calm water in free motion with an initial velocity. A Cartesian system $O-x y$ moving translationally with the body is defined, in which the origin $O$ is fixed at the wedge tip, $x$-axis points horizontally and $y$-axis points vertically upwards. The horizontal and vertical velocities of $O$ which is taken as the rotational centre are denoted as $U$ and $W$, respectively, and $W$ is positive when the body moves downwards. The angular velocity about $O$ is denoted as $\Omega$. At $t=0, U_{0}, V_{0}$ and $\Omega_{0}$ are used to denote the initial velocities. $\theta$ is the heel angle measured from the symmetry line of the wedge to $y$ axis, and $\theta_{0}$ is its initial value at the moment of water entry. $\gamma_{0}$ is half of the inner angle of the wedge.

Based on the these definitions, the deadrise angles $\gamma_{1}$ and $\gamma_{2}$ on right and left hand sides can respectively be obtained from the following relationships

$$
\gamma_{1}=\frac{\pi}{2}+\theta-\gamma_{0}, \gamma_{2}=\frac{\pi}{2}-\theta-\gamma_{0}
$$

The vertical distance $s$ and horizontal distance $l$ that the wedge has travelled, and the heel angle $\theta$ can respectively be obtained through

$$
\dot{s}(t)=W(t), \quad \dot{l}(t)=U(t), \quad \dot{\theta}(t)=\Omega(t)
$$

\subsection{Mathematical model}

The fluid domain contains two separate ones. One of these domains, the inner one, is the fluid inside the tank, and the other one, the outer one, is the open water. When the fluid is assumed to be incompressible and inviscid, and the flow to be irrotational, the velocity potential $\phi_{i}$ whose 
gradient is equal to the fluid velocity can be introduced, which satisfies the Laplace equation in the whole fluid domain

$$
\nabla^{2} \phi_{i}=0, \quad(i=1,2)
$$

in which the subscripts 1 and 2 correspond to the inner and outer domains respectively. In the coordinate system $O-x y$, the dynamic and kinematic boundary conditions on the instantaneous free surface $S_{\mathrm{F}}$ in Lagrangian form can respectively be written as

$$
\begin{gathered}
\frac{D \phi_{i}}{D t}=\frac{1}{2}\left|\nabla \phi_{i}\right|^{2}-g(y-s) \\
\frac{D x}{D t}=\frac{\partial \phi_{i}}{\partial x}-U, \frac{D y}{D t}=\frac{\partial \phi_{i}}{\partial y}+W
\end{gathered}
$$

where $D / D t$ is the full derivative following the fluid particle, and $(x, y, \varphi)$ for the fluid particle can then be tracked through a time integration scheme. $U$ and $W$ in Eq. (5) are due to the fact that $O-x y$ is a moving system. On the wetted surface of body $S_{0}$, the impermeable boundary condition takes the form as

$$
\frac{\partial \phi_{i}}{\partial n}=(U-\Omega y) n_{x}+(-W+\Omega x) n_{y}
$$

where $\mathbf{n}=\left(n_{x}, n_{y}\right)$ is the normal vector on the body surface pointing out of the corresponding fluid domain, and $\mathbf{x}=(x, y)$ is the position vector relative to the rotational centre $O$. The negative sign before $W$ is due to the fact that $W$ is positive when it is downwards. Far away from the body, the disturbance is assumed to have sufficiently diminished, and the far field boundary condition for open water can therefore be written as

$$
\nabla \phi_{2}=0
$$

At $t=0$, the initial condition can be written as

$$
y_{i}(x, t=0)=H_{i}, \phi_{i}\left(x, H_{i}, t=0\right)=0
$$

where $H_{1}$ is the depth of water inside the wedge at $t=0$, and $H_{2}=0$ reflects the calm water surface in the open domain.

At the initial stage of water entry, there is only a tiny part of body in water. In order to ensure sufficient numerical accuracy for the solution in the outer domain, we adopt the method in Wu et al. [9] and Sun et al. [11]. A stretched coordinate system $O-\alpha \beta$ is defined as

$$
\phi_{2}(x, y, t)=s \varphi(\alpha, \beta, t), \alpha=x / s, \beta=y / s
$$

in which $s$ is given in Eq. (2). The body surface boundary condition for $\varphi$ retains the same form as that in Eq. (6)

$$
\frac{\partial \varphi}{\partial n}=(U-s \Omega \beta) n_{\alpha}+(-W+s \Omega \alpha) n_{\beta}
$$

The free surface boundary conditions can be written as

$$
\frac{D(s \varphi)}{D t}=\frac{1}{2}\left(\varphi_{\alpha}^{2}+\varphi_{\beta}^{2}\right)-g(s \beta-s)
$$




$$
\frac{D(s \alpha)}{D t}=\varphi_{\alpha}-U, \frac{D(s \beta)}{D t}=\varphi_{\beta}+W
$$

\subsection{The boundary element method}

The Laplace equation in the fluid domain can be converted into the integral equation over the whole boundary

$$
A(p) \phi(p)=\int\left(\phi(q) \frac{\partial \ln r_{\mathrm{pq}}}{\partial n_{\mathrm{q}}}-\ln r_{\mathrm{pq}} \frac{\partial \phi(q)}{\partial n_{\mathrm{q}}}\right) d l_{\mathrm{q}}
$$

where $A(p)$ is the solid angle of boundaries at the point $p$ and $r_{\mathrm{pq}}$ is the distance from the field point $p$ to the source point $q$. $\phi$ in the equation stands for either $\phi_{1}$ or $\phi_{2}$. Straight line elements, with variables being assumed to vary linearly within each element, are distributed along the boundary

(a)

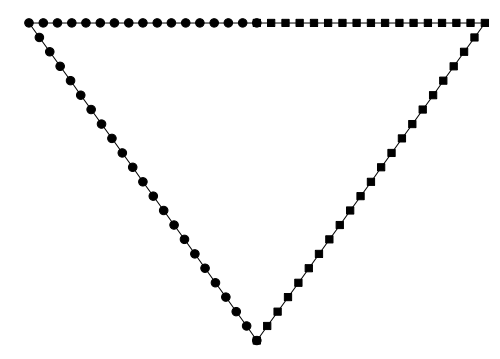

(b)

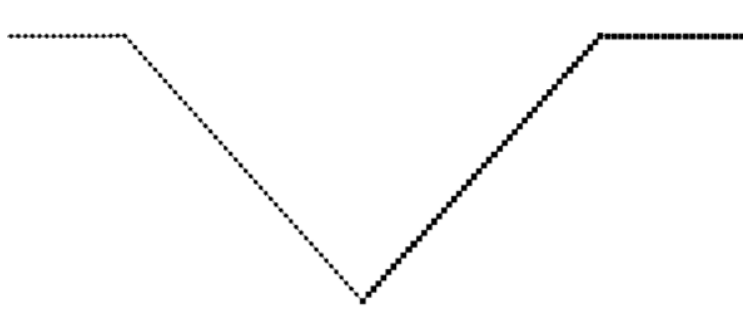

Fig. 2. The discretization of boundary for (a) the inner domain in $(x, y)$ and (b) outer domain in $(\alpha, \beta)$ near the wedge at the initial moment of computation.

Fig. 2 provides a typical discretization of the boundaries for both inner and outer domains. These two domains are fully separated. Their elements on the body surface do not coincide and are distributed for the consideration of their corresponding domains. To perform integration over each element in Eq. (13), we define

$$
\mathbf{r}=x \mathbf{i}+y \mathbf{j}=\sum_{i=1}^{2} h^{i}(u) \cdot \mathbf{r}^{i}, \quad \phi=\sum_{i=1}^{2} h^{i}(u) \cdot \phi^{i}
$$

in which $\mathbf{r}$ is the position vector from the origin with $\mathbf{i}$ and $\mathbf{j}$ being the unit vectors in the $x$ and $y$ directions. When the stretched system is used, $x$ and $y$ will be replaced by $\alpha$ and $\beta$ respectively. Linear shape functions $h^{i}(u)$ are used, which can be written in the following form

$$
h^{1}(u)=1-u, h^{2}(u)=u
$$

where $0 \leq u \leq 1$, when point $q$ moves from one end of the element to the other. Substituting Eqs. (14) and (15) into (13), we have

$$
A(p) \phi(p)=\sum_{j=1}^{N_{\mathrm{e}}} \int_{0}^{1} \ln r_{\mathrm{pq}} \sum_{i=1}^{2} h^{i}(u) \frac{\partial \phi_{j}^{i}(q)}{\partial n} l d u-\sum_{j=1}^{N_{\mathrm{e}}} \int_{0}^{1} \frac{\partial \ln r_{\mathrm{pq}}}{\partial \mathrm{n}} \sum_{i=1}^{2} h^{i}(u) \phi_{j}^{i}(q) l d u
$$

where $N_{e}$ is the total number of elements. The integrations within each element can be obtained through the analytical solution from $\mathrm{Lu}, \mathrm{He} \& \mathrm{Wu}$ [28]. Writing Eq. (16) in the matrix form, we have 


$$
[H]_{N_{\mathrm{d}} \times N_{\mathrm{d}}}[\phi]_{N_{\mathrm{d}}}=[G]_{N_{\mathrm{d}} \times N_{\mathrm{d}}}\left[\frac{\partial \phi}{\partial n}\right]_{N_{\mathrm{d}}}
$$

where $N_{\mathrm{d}}$ is the total number of nodes over the whole boundary, and the matrixes of $[H]$ and $[G]$ have the coefficients obtained from the integrations of $\frac{\partial \ln r_{\mathrm{pq}}}{\partial \mathrm{n}}$ and $\ln r_{\mathrm{pq}}$ respectively.

Based on the boundary conditions, the normal derivative of the potential on the solid surface and the potential on the free surface are known at each time step. They can both be moved to the right hand side, while the unknowns are moved to the left. Eq. (17) can then be transformed as

$$
\left[\begin{array}{ll}
H_{b b} & -G_{b f} \\
H_{f b} & -G_{f f}
\end{array}\right] \cdot\left[\begin{array}{c}
\phi_{b} \\
\phi_{n f}
\end{array}\right]=\left[\begin{array}{ll}
G_{b b} & -H_{b f} \\
G_{f b} & -H_{f f}
\end{array}\right] \cdot\left[\begin{array}{c}
\phi_{n b} \\
\phi_{f}
\end{array}\right]
$$

where the subscript $b$ denotes the boundary including the solid surface and the far field surface while $f$ denotes the free surface. When Eq. (18) is used in the inner domain, there will be only the wedge surface and the free surface.

\subsection{The pressure}

Based on the Bernoulli equation, and assuming that the pressures on the free surface both inside and outside the tank are atmospheric, which can be taken as zero without loss of generality, the pressure in both fluid domains can be written as

$$
p_{i}=-\rho_{i}\left[\phi_{i t}+\frac{1}{2}\left|\nabla \phi_{i}\right|^{2}+g(y-s)\right]
$$

where $\rho_{i}(i=1,2)$ are the liquid densities inside and outside the tank respectively. The velocity potential $\phi_{i}$ can be solved at each time step through the numerical scheme in the previous section, from which its gradient $\nabla \phi_{i}$ can be obtained. However the temporal derivative of potential $\phi_{i t}$ is still unknown explicitly. To deal with this problem, the auxiliary function method [27] is adopted here. We notice that $\phi_{i t}$ also satisfies the Laplace's function in the fluid domain. The normal derivative of $\phi_{i t}$ on the body surface can be written as [29]

$$
\frac{\partial \phi_{i t}}{\partial n}=(\dot{\mathbf{U}}+\dot{\boldsymbol{\Omega}} \times \mathbf{x}) \cdot \mathbf{n}-\mathbf{U} \cdot \frac{\partial \nabla \phi_{i}}{\partial n}+\mathbf{\Omega} \cdot \frac{\partial}{\partial n}\left[\mathbf{x} \times\left(\mathbf{U}-\nabla \phi_{i}\right)\right]
$$

where $\mathbf{U}=U \mathbf{i}+W \mathbf{j}, \quad \mathbf{\Omega}=\Omega \mathbf{i} \times \mathbf{j}$, and the over dot means the temporal derivative. A particular attention should be paid to accelerations in Eq. (20), which are unknown before the force is found. To decouple their mutual dependence, we define

$$
\phi_{i t}=\chi_{i 0}+\dot{U} \chi_{i 1}+\dot{W} \chi_{i 2}+\dot{\Omega} \chi_{i 3}-\mathbf{U} \cdot \nabla \phi_{i}+\boldsymbol{\Omega} \cdot\left[\mathbf{x} \times\left(\mathbf{U}-\nabla \phi_{i}\right)\right]
$$

Here $\chi_{i j}(i=1,2 ; j=0 \cdots 3)$ satisfy the Laplace's equation. The body surface boundary conditions for the auxiliary functions can be written as 


$$
\frac{\partial \chi_{i j}}{\partial n}=n_{j}, n_{0}=0, n_{1}=n_{x}, n_{2}=n_{y}, n_{3}=\left(x n_{y}-y n_{x}\right),(i=1,2, j=0, \cdots 3)
$$

Considering zero pressure on the free surface in Eq. (19), we have

$$
\begin{gathered}
\chi_{i 0}=-\left[\frac{1}{2}\left|\nabla \phi_{i}\right|^{2}+g(y-s)\right]+U \phi_{i x}-W \phi_{i y}-\Omega\left[x\left(-W-\phi_{i y}\right)-y\left(U-\phi_{i x}\right)\right] \\
\chi_{i j}=0,(i=1,2, j=1,2,3)
\end{gathered}
$$

At the far field of the outer domain, the disturbance to the fluid is assumed to have sufficiently diminished, and thus $\nabla \phi=0, \nabla \phi_{t}=0$. We have from Eq. (20)

$$
\begin{gathered}
\frac{\partial \chi_{20}}{\partial n}=\Omega\left(n_{x} W+n_{y} U\right) \\
\frac{\partial \chi_{2 i}}{\partial n}=0,(i=1,2,3)
\end{gathered}
$$

Here $\mathbf{n}=\left(n_{x}, n_{y}\right)$ in Eq. (25) is the normal of the far filed boundary. The solution of above auxiliary functions can be found through the boundary element method in Sec. 2.3. Based on Eqs. (19) and (20), we can find that only the accelerations are unknown in $\phi_{i t}$, which will be solved through the following coupled equation of motion. Particularly, different from previous water entry problems, the fluid load comes not only from the outer domain, but also from the fluid within the liquid tank, which may have some major effects.

\subsection{Equation of motion}

The equation of motion for a wedge tank filled with liquid entering water can be written as

$$
\left[\mathbf{M}_{0}\right][\mathbf{A}]=\left[\mathbf{F}_{1}\right]+\left[\mathbf{F}_{2}\right]+\left[\mathbf{F}_{\mathrm{G}}\right]
$$

where

$$
\left[\mathbf{M}_{0}\right]=\left[\begin{array}{ccc}
m_{0} & 0 & -m_{0} l_{c 0} \cos \theta \\
0 & m_{0} & -m_{0} l_{c 0} \sin \theta \\
-m_{0} l_{c 0} \cos \theta & -m_{0} l_{c 0} \sin \theta & I_{0}
\end{array}\right]
$$

is the mass matrix for the wedge shell with $m_{0}$ being the mass, and $I_{0}$ being the rotational inertia and and $l_{c 0}$ is the distance between the mass centre on the symmetry line and the rotational centre. [A] in Eq. (28) is a column of accelerations, which can be solved through Gaussian elimination method for Eq. (27). $\left[\mathbf{F}_{1}\right],\left[\mathbf{F}_{2}\right]$ and $\left[\mathbf{F}_{\mathrm{G}}\right]$ in the equations are the internal and external hydrodynamic forces, and the gravitational force of the body, respectively. The first two forces can be obtained through the integration of pressures along the inner and outer wetted surfaces, $S_{1}$ and $S_{2}$, of the body, respectively. Thus we have 


$$
\mathbf{F}_{i}=-\rho_{i} \int_{S_{i}}\left\{\chi_{i 0}+\dot{U} \chi_{i 1}+\dot{W} \chi_{i 2}+\dot{\Omega} \chi_{i 3}-\mathbf{U} \cdot \nabla \phi_{i}+\boldsymbol{\Omega} \cdot\left[\mathbf{x} \times\left(\mathbf{U}-\nabla \phi_{i}\right)\right]+\frac{1}{2}\left|\nabla \phi_{i}\right|^{2}+g(y-s)\right\} \mathbf{n} d s
$$

Substituting Eq. (29) into (27), and moving the acceleration terms to the left hand side, we obtain

$$
\left(\left[\mathbf{M}_{0}\right]+\left[\mathbf{C}_{1}\right]+\left[\mathbf{C}_{2}\right]\right)[\mathbf{A}]=\left[\mathbf{F}_{1}^{\prime}\right]+\left[\mathbf{F}_{2}^{\prime}\right]+\left[\mathbf{F}_{\mathrm{G}}\right]
$$

in which

$$
C_{i j k}=\rho_{i} \int_{S_{i}} \chi_{i j} n_{k} d s
$$

are the added masses corresponding to internal and external fluids respectively, and

$$
\mathbf{F}_{i}^{\prime}=-\rho_{i} \int_{S_{i}}\left\{\chi_{i 0}-\mathbf{U} \cdot \nabla \phi_{i}+\mathbf{\Omega} \cdot\left[\mathbf{x} \times\left(\mathbf{U}-\nabla \phi_{i}\right)\right]+\frac{1}{2}\left|\nabla \phi_{i}\right|^{2}+g(y-s)\right\} \mathbf{n} d s
$$

Eq. (30) shows that while the potentials inside and outside the wedge can be solved independently, the fluid flows are still coupled through the body motion. Therefore the flows in the two subdomains are not entirely independent.

An approximation is to treat inner fluid stationary if its motion is small. $\phi_{1}$ can be taken as zero and $\left[\mathbf{F}_{1}^{\prime}\right]$ contains only the hydrostatic force which is due to the weight of the stationary liquid. It may be interesting to compare this with the case in which the inner liquid has become a solid mass such as a frozen ice block. In such a case, $\left[\mathbf{F}_{1}^{\prime}\right]$ is due to the ice block weight, which is the same as that of the stationary liquid. However $\left[\mathbf{C}_{1}\right]$ should be replaced by mass matrix $\left[\mathbf{M}_{1}\right]$ of the ice block. Similar to $\left[\mathbf{M}_{0}\right]$, the mass matrix for $\left[\mathbf{M}_{1}\right]$ can be written as

$$
\left[\mathbf{M}_{1}\right]=\left[\begin{array}{ccc}
m_{1} & 0 & -m_{1} l_{c 1} \cos \left(\theta-\theta_{0}+\theta_{1}\right) \\
0 & m_{1} & -m_{1} l_{c 1} \sin \left(\theta-\theta_{0}+\theta_{1}\right) \\
-m_{1} l_{c 1} \cos \left(\theta-\theta_{0}+\theta_{1}\right) & -m_{i} l_{c i} \sin \left(\theta-\theta_{0}+\theta_{1}\right) & I_{1}
\end{array}\right]
$$

in which $m_{1}$ and $I_{1}$ denote the mass and rotational inertia of the ice block respectively, and $l_{c 1}$ denotes the distance between the mass centre and rotational centre $O$. It should be noted that the angle $\theta_{1}$, which is the angle between the line linking the mass centre of the ice block to $O$ and $y$ axis, is different from $\theta_{0}$. Intuitively, stationary liquid may seem to be the same as a frozen ice block. However, Eq. (30) shows that the difference is between $\left[\mathbf{C}_{1}\right]$ and $\left[\mathbf{M}_{1}\right]$. In fact the former is due to the fact that the acceleration at each point inside the stationary liquid is different, while in the latter, the acceleration at each point can be decomposed into two translational accelerations and one rotational acceleration, as common in the rigid body problem. Therefore these two cases are not identical. An exceptional case is when there is vertical motion only, in which the free surface will remain stationary relative to the tank. In such a case, $\chi_{12}=y-H_{1}$ and $c_{122}=m_{1}$. The motions with the liquid and ice will be the same.

\section{Numerical results and discussions}




\subsection{Convergence study and comparison}

In order to test the numerical stability and accuracy of the methodology in the paper, we first consider the case of the wedge tank entering into water freely with a given initial velocity, $U=0$, $W=5 \mathrm{~ms}^{-1}$, and $\Omega=1 \mathrm{~s}^{-1} \cdot \gamma_{0}$ and $\theta_{0}$ are set as $\pi / 4$ and 0 respectively, the depth of water inside the tank is set as $H_{1}=0.5 \mathrm{~m}$, and the water densities are set as $\rho_{1}=\rho_{2}=1025 \mathrm{kgm}^{-3}$. The mass and rotational inertia of wedge shell are both set as zero, or $\left[\mathbf{M}_{0}\right]=0$ in Eq. (30). To start the simulation, a tiny part of body is put into water at initial time step and the distance from the tip of the body to the still water surface in the physical system is denoted as $s_{0}$, or the initial value of $s$. It should be noted that no matter how small $s_{0}$ is, the vertical distance between the tip and the outer still water surface is always unit in the stretched system. In this simulation, $s_{0}$ is set as $H_{1} / 500$. The length and the depth of the outer computational domain are respectively set as $\alpha= \pm 50$ and $\beta=-50$ in the stretched system. Unequal elements are distributed along the fluid boundary. The smallest elements of length $l_{\mathrm{m}}$ are used on the body surface and the part of the outer free surface, or from the intersection with the body surface to a point $Q$ whose perpendicular distance in the stretched system to the surface of wedge is 1 . Beyond the point $Q$, the size of the element increases gradually at a fixed ratio $\delta$, but is limited by a maximum. For convergence study, we now set $l_{m}$ as 0.02 and 0.03 respectively, and $\delta$ as 1.02 . It should be noted that $l_{m}$ is nondimensional in the stretched system of the outer domain. In the inner domain, the elements of equal size are distributed both on internal free surface and body surface, and $l_{m} H_{1}$ is used in the dimensional sense. The time histories of accelerations with these two different meshes are shown in Fig. 3. A good agreement can be seen in the figure, which means that the results are mesh independent. The time step is set as $d t=s_{0} /(\mu W)$, with an upper limit of $d t=2 l_{m} /\left(\mu V_{\max }\right)$, in

which $\mu$ is a coefficient, and $V_{\max }$ is the largest magnitude of velocity on the free surface. The advancement of $s$ can be well controlled through the first equation, and the requirement that the fluid particle cannot travel more than one element size within each time step is achieved by the second equation. Fig. 4 gives the comparison of velocity histories of the wedge between $\mu=10$ and $\mu=20$. It can be seen that the two curves are virtually coincident, which shows that the results have converged with the time step. Unless it is specified, in the following simulations, the minimal element length $l_{m}=0.03$, the element size increase ratio $\delta=1.02$, time step coefficient $\mu=10$, and the mass of the wedge shell is neglected. 

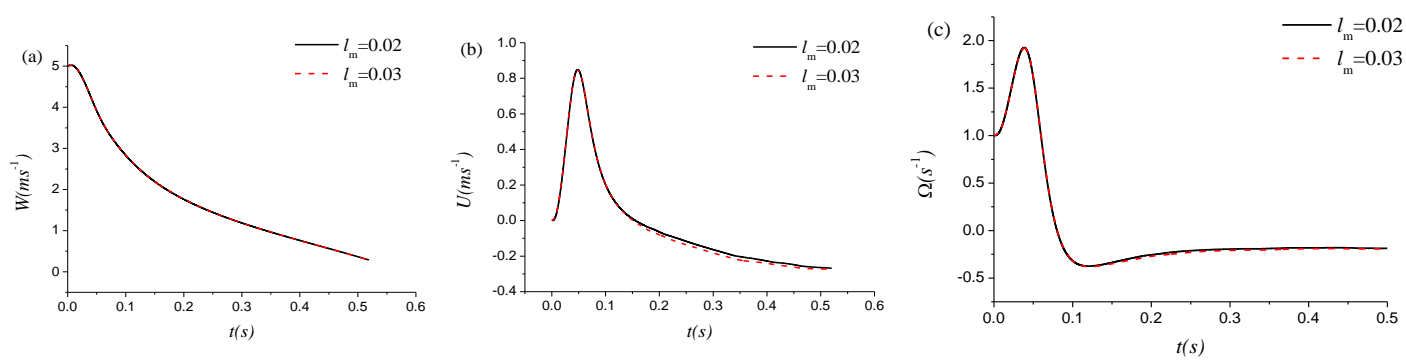

Fig. 3. Mesh convergence study: (a) vertical, (b) horizontal and (c) rotational accelerations.
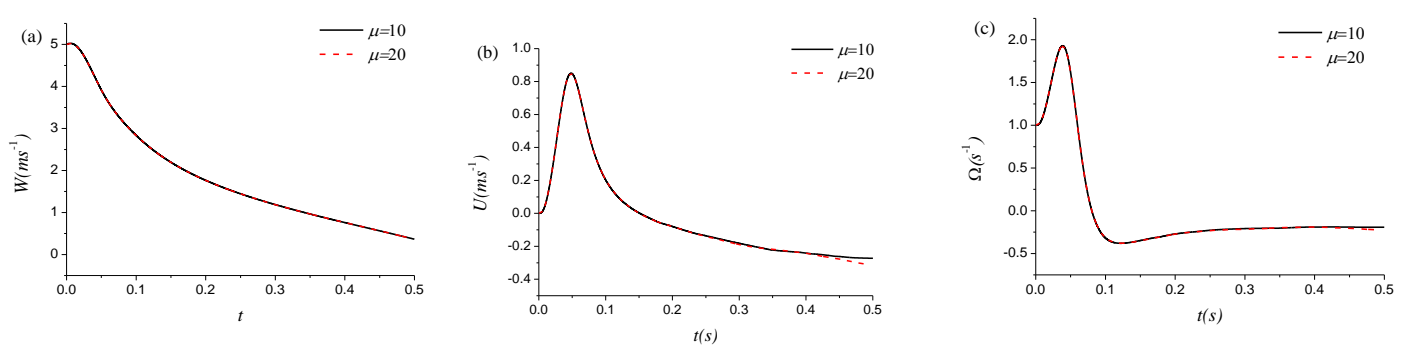

Fig. 4. Time step convergence study: (a) vertical, (b) horizontal and (c) rotational accelerations.

Fig. 5 gives the comparison between the results from the present simulation and those from the experiment and numerical computation of $\mathrm{Wu}$ et al. [9]. The water inside the tank is treated as an ice block with mass $m_{1}=13.522 \mathrm{kgm}^{-1}$ and $30.188 \mathrm{kgm}^{-1}$ respectively, in two different simulations. The density distribution of the ice block is assumed to non-uniform to match the rotational inertia $I_{1}=45 \mathrm{kgm}^{2}$ in Wu et al. [9]. $g_{e}$ is the real acceleration of body before touching water, which is smaller than the gravity acceleration $g$ due to the effect of frictional force in the experiment. In the numerical simulation, the gravity effect for free surface, or the last term in Eqs. (4) and (11), is neglected, as in the numerical simulation of Wu et al. [9]. Good agreement is clearly evident. This proves the rational of the present model and the accuracy of the numerical procedure.
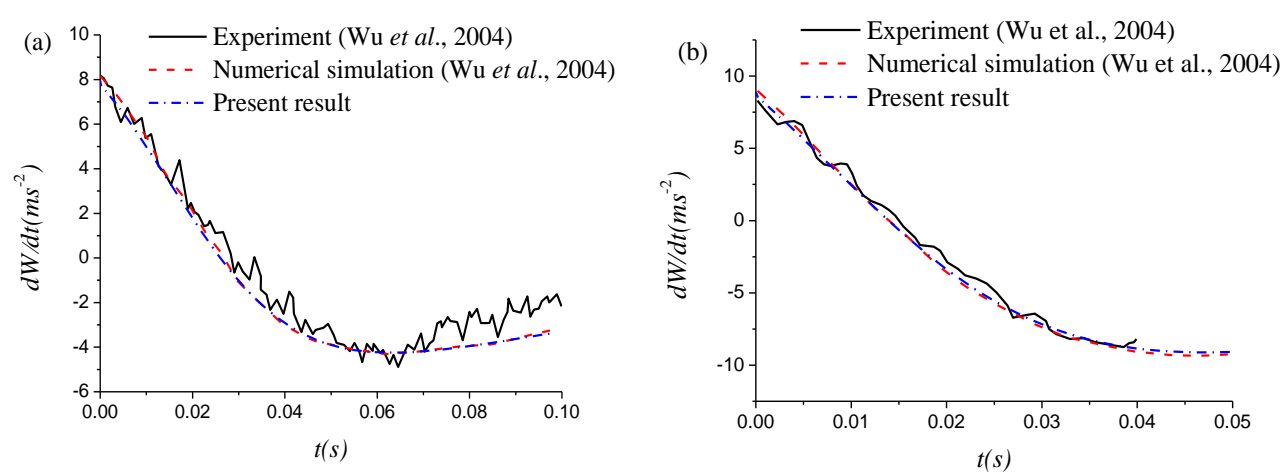

Fig. 5. The vertical acceleration at $\gamma_{0}=\pi / 4, \theta_{0}=\theta_{1}=0$ and $I_{1}=45 \mathrm{kgm}^{2}$ : (a)

$$
\begin{aligned}
& g_{e}=8.0062 \mathrm{~ms}^{-2}, \quad m_{1}=13.522 \mathrm{kgm}^{-1}, W=0.95623 \mathrm{~ms}^{-1} \quad, \quad \text { (b) } g_{e}=8.9716 \mathrm{~ms}^{-2}, \\
& m_{1}=30.188 \mathrm{kgm}^{-1}, W_{0}=1.69673 \mathrm{~ms}^{-1} .
\end{aligned}
$$




\subsection{Water entry of a wedge tank with internal water of different depth $H_{1}$}

For the liquid contained in a container, there will be usually sloshing motion. The sloshing motion is very much affected by the natural frequencies of the container, at which the fluid will have an oscillatory motion once it has some initial disturbance. This motion will not diminish when the viscosity of the liquid has been ignored. For a wedge with $\gamma_{0}=\pi / 4$, Table 1 gives the natural

periods $T_{N}$ of the sloshing motion inside the wedge tank in terms of $T_{N} \sqrt{\mathrm{g} / H_{1}}$ based on the equation in Lamb [30], which is independent of $H_{1}$ itself.

\begin{tabular}{|l|l|l|l|l|l|}
\hline \multicolumn{1}{|c|}{ Order } & & & & \\
$T_{N} \sqrt{\mathrm{g} / H_{1}}$ & $1^{\text {st }}$ & $2^{\text {nd }}$ & $3^{\text {rd }}$ & $4^{\text {th }}$ & $5^{\text {th }}$ \\
\hline Symmetric modes & 4.12 & 2.68 & 2.14 & 1.83 & 1.63 \\
\hline Asymmetric modes & 3.17 & 2.36 & 1.96 & 1.72 & 1.55 \\
\hline
\end{tabular}

Table 1. Natural periods of sloshing motion inside the wedge tank with $\gamma_{0}=\pi / 4$

When a wedge tank filled with liquid enters into water, the liquid motion in the tank may exert noticeable effect on the motion of body, and then the motion of the body will further affect the slamming pressure along the outer surface of the wedge. They are therefore entirely coupled. To study the mutual dependence among all these three, the wedge tank with water of depth $H_{1}$ is released into calm water with a given initial velocity and subsequently in free motion. In this and following sections, the initial vertical velocity of the wedge $W_{0}$, the acceleration due to gravity $g$, the external water density $\rho_{2}$ are used for nondimensionalisation, and the superscript ' is used for the nondimensionalized parameters.

Fig. 6 provides the time histories of velocities of the wedge tank with $H_{1}^{\prime}$ equal to $0.196,0.392$ and 0.784 respectively, and $\rho_{1}^{\prime}$ is set as 1 , assuming that the internal liquid is the same as the external one. The initial velocities are set as $U_{0}^{\prime}=0, W_{0}^{\prime}=1$ and $\Omega_{0}^{\prime}=1.02$. The results are compared with those of ice of the same weight to see the effect of the liquid motion. In the latter case, the added mass matrix $\left[\mathbf{C}_{1}\right]$ of the liquid is replaced by the mass matrix of ice block $\left[\mathbf{M}_{1}\right]$, and the fluid force is changed to the force due to ice weight. It ought to be noticed that there are two time scales corresponding to water entry and sloshing motion inside the tank respectively. In the former it is measured in the form of $t^{\prime}=t g / W_{0}$. In this time scale the wedge of constant speed $W_{0}^{\prime}$ will travel the same nondimenisonal distance at the same nondimensional time. In the latter time scale for sloshing, the oscillation is measured by $t_{2}^{\prime}=t \sqrt{g / H_{1}}=t^{\prime} \sqrt{1 / H_{1}^{\prime}}$. The period $T_{2}^{\prime}$ measured in $t_{2}^{\prime}$ is the same for all water depth $H_{1}$, as shown in Table 1 . Thus for large $H_{1}^{\prime}$, to reach the same period of $T_{2}^{\prime}, t^{\prime}$ has to be larger. In other words, at larger $H_{1}^{\prime}$, it requires a larger impact time to excite the resonant motion of the internal fluid. Before that, the effect of the liquid is mainly due to its initial motion rather than the full oscillatory sloshing motion, especially at larger 
$H_{1}^{\prime}$. Also the effect of $H_{1}^{\prime}$ is not just through the natural frequency, but also though the weight.

The vertical velocity in Fig. 6(a) increases initially, and then it reaches a peak. This is mainly attributed to the mutual dependence between the fluid load and body motion. At the initial stage, the wetted area and impact force need time to increase, and $W^{\prime}$ increases because of acceleration due to the inner fluid mass. For the larger $H_{1}^{\prime}$ with larger inner fluid mass, the acceleration period will be longer and the peak of $W^{\prime}$ will be larger. As time increases, the external fluid load will balance with the fluid load inside the tank. After that, acceleration changes to deceleration, and the vertical velocity decreases. For the case of $H_{1}^{\prime}=0.784$, as its $W^{\prime}$ reaches a larger peak, it generates a larger impact force. It then has a larger deceleration. As $W^{\prime}$ decreases rapidly, so does the impact force. $W^{\prime}$ then reaches a trough around $t^{\prime} \approx 0.32$. Beyond that $W^{\prime}$ increases again. From the result in Table 1, the lowest sloshing period is much larger than the whole duration of water entry, and this implies that the oscillation of vertical velocity in Fig. 6(a) is mainly due to the variation of impact force rather than the sloshing motion of the internal water.

At a given anticlockwise angular velocity $\Omega_{0}^{\prime}, \Omega^{\prime}$ increases initially, mainly due to that the additional anticlockwise rotational moment as the mass centre of internal water moves leftwards. During this process, the impact force on the left hand side increases, because the deadrise angle on the left hand side becomes smaller. This will increase the clockwise moment and make the rate of the increase of $\Omega^{\prime}$ decline. When the impact moment balances the moment due to internal one, the angular velocity curve reaches a peak, which occurs at $t^{\prime} \approx 0.32$ for $H_{1}^{\prime}=0.784$. It is interesting to see that the time is close to that of the trough in Fig. 6(a) for the vertical velocity. After the peak, the acceleration changes to deceleration, and eventually the tank begins to rotate in the opposite direction. The horizontal velocity in Fig. 6(b) increases initially and then decreases. This is mainly due to the increase of impact pressure on the left hand side at the earlier stage. As the anticlockwise motion continues, the deadrise angle on the left hand side becomes smaller and the one on the right becomes larger. The horizontal component of the force on the left becomes much smaller than its vertical component, while it is opposite on the right. As a result, the total horizontal force changes its direction and the horizontal velocity begins to decline.

As the fluid inside the tank moves, different fluid particles would move with different accelerations, which may lead to that the total inertial force becomes different from that of a solid ice with the same weight. It is then possible that the inertia force of water in the vertical direction is smaller than that of ice, and the liquid wedge tank is therefore easier to decelerate and accelerate. Thus in Fig. 6(a), the vertical velocity of the tank with water varies more rapidly than that with the ice during either the deceleration period or the acceleration period. Similarly in horizontal direction or rotational direction, the motion of fluid inside the tank makes the tanks far more responsive to the impact force. 

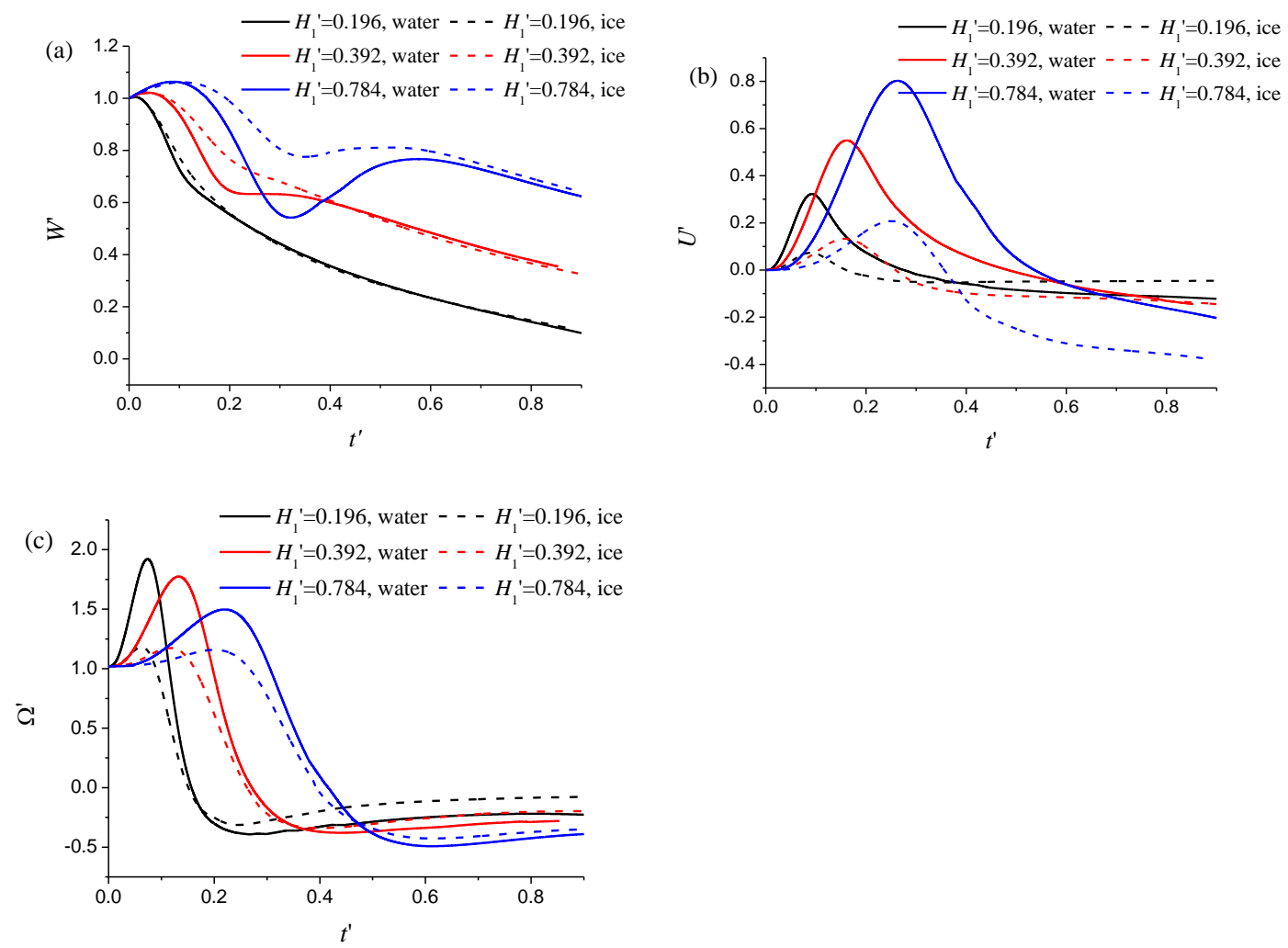

Fig. 6. Velocities versus time $t^{\prime}$ for water entry of a wedge tank $\left(\gamma_{0}=\pi / 4, \theta_{0}=0\right)$ with water or ice of different depth: (a) vertical, (b) horizontal and (c) rotational.

\subsection{Water entry of a wedge tank with internal water of different densities $\rho_{1}^{\prime}$}

The fluid inside the wedge tank may have different densities. A typical example on a ship is the liquefied natural gas, or a ship container with a mixture of water and sand, whose physical characteristics are similar to fluid, but the density is much larger. Fig. 7 shows the time histories of velocities of the wedge tank with $\rho_{1}^{\prime}$ equal to 1,2 and 3 respectively, in which $H_{1}^{\prime}$ is set as 0.392 and the initial velocities as $U_{0}^{\prime}=0, W_{0}^{\prime}=1$ and $\Omega_{0}^{\prime}=1.02$. As discussed above, the vertical velocity of the tank increases due to gravitational acceleration at the initial stage of impact. When $\rho_{1}^{\prime}$ is larger, the weight is larger relative to the impact force. The duration of acceleration is longer, and the first peak point of the vertical velocity moves rightwards in the time history curve. After the peak, the velocity decreases due to the increased impact pressure and wetted area. It will increase again due to the decrease of impact pressure, and then reach the second peak. The prominent difference between the cases of water and ice block is the much more obvious trough in the former in Fig. 7(a), which is due to the fact that the tank with fluid is more responsive to the change of impact force. For the same reason, the peaks and troughs of the horizontal and rotational velocities in the case of tank with liquid are much larger. It is interesting to see that as time increases the results of the cases of both liquid and ice tend to be very close. As it can be seen from the results, the accelerations tend to constant as time increases. The accelerations of most fluid particles inside the tank also tend to the same in such a case, except that within the small area near the intersection point between free surface and body surface, where the fluid particle may move with different accelerations. Accordingly, the free surface tends to move upwards along the tank wall. It is of 
interest to see that the troughs at different $\rho_{1}^{\prime}$ occur almost at the same time, and it is similar in the subsequent peaks. Similar patterns can be seen in Fig. 7(b) and (c). This is because when the fluid depth inside the tank is the same, the natural periods at different densities are the same and the internal liquid motion follows a similar time scale. Also at larger $\rho_{1}^{\prime}$ the magnitude of vertical velocity is larger because of larger weight, while the magnitudes of horizontal velocity and rotational velocity become smaller due to larger mass.
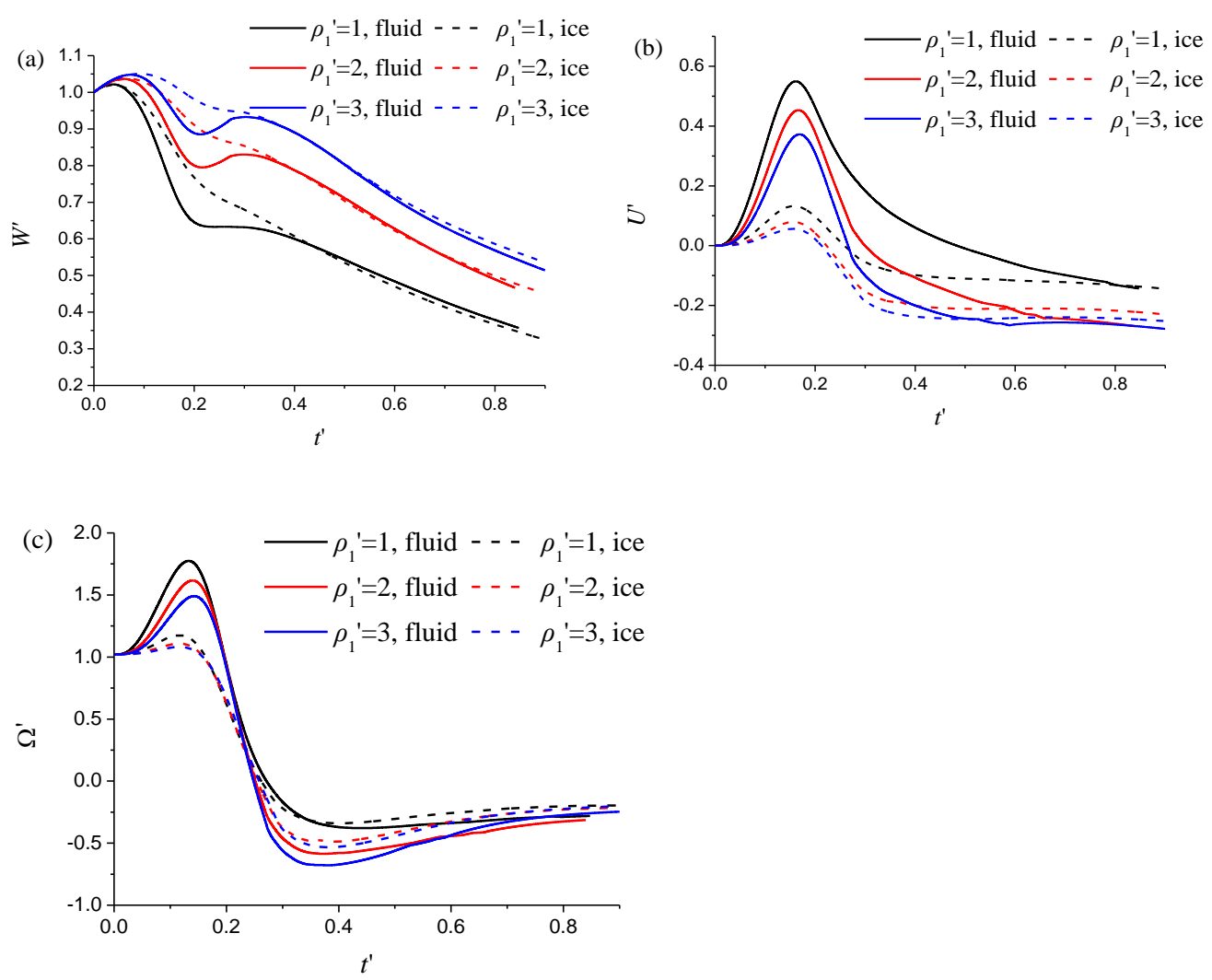

Fig. 7. Velocities versus time $t^{\prime}$ for the water entry of a wedge tank $\left(\gamma_{0}=\pi / 4, \theta_{0}=0\right.$, $H_{1}^{\prime}=0.392$ ) with internal liquid of different densities: (a) vertical, (b) horizontal and (c) rotational. 3.4 Water entry of a wedge tank with different initial angular velocities

Fig. 8 provides the time histories of accelerations and velocities of the wedge tank with $H_{1}^{\prime}=0.196$ and $\rho_{1}^{\prime}=1$. The initial rotating velocities $\Omega_{0}^{\prime}$ are set as $0,0.51$ and 1.02 respectively, with $U_{0}^{\prime}=0$. In Fig. 8(a), it can be seen that the accelerations at different $\Omega_{0}^{\prime}$ all decrease and become deceleration very rapidly, and the rate of change is larger at larger $\Omega_{0}^{\prime}$. They reach a different trough around the same time $t^{\prime}$, which is not too much affected by $\Omega_{0}^{\prime}$. After the trough, the acceleration increases rapidly again initially and then the rate of increase declines. In fact, the vertical acceleration is mainly attributed to the fluid force exerted on the outer surface of the wedge. The forces increase both with fluid pressure which mainly depends on the entry speed, and the wetted area which increases with $s^{\prime}$. At very small $t^{\prime}$, the velocity $W^{\prime}$ increases because 
of the acceleration motion. Very rapidly, the hydrodynamic force will increase, which will reduce the acceleration. As the force increases further, the body motion enters deceleration. All these happen in a very short period of time, as shown in Figs. 8(a) and 8(b). As $t^{\prime}$ further increases, the wetted surface increases while $W^{\prime}$ tends to zero. It is expected that the body may bounce upwards eventually. This is however beyond the scope of the current study as the focus of the work is on water entry. For the case of $\Omega_{0}^{\prime}=0$, the inner liquid is stationary and the results coincide with those corresponding to ice block. As discussed at the last paragraph of Sec. 3.2, generally the motions with the liquid and ice will be different, even when the liquid is stationary, but an exception is when $\Omega^{\prime}=0$ and $U^{\prime}=0$, which is reflected in the results here. As $\Omega_{0}^{\prime}$ increases, the trough of the acceleration curve becomes lower, which is even much lower for the case with water, as shown in Figs. 8(c) and (e). However, the difference between the acceleration curves is mainly in a small region near the trough. As a result the curves for $W^{\prime}$ are all very close. This indicates that the external vertical forces are similar in these cases. Figs. 8(e) and (f) show a larger $\Omega_{0}^{\prime}$ leads to a larger rotational acceleration in the same direction at the initial stage. This is because the rotational moment due to external impact force is small at the initial stage and the difference in the angular acceleration is principally caused by the motion of the inner liquid.
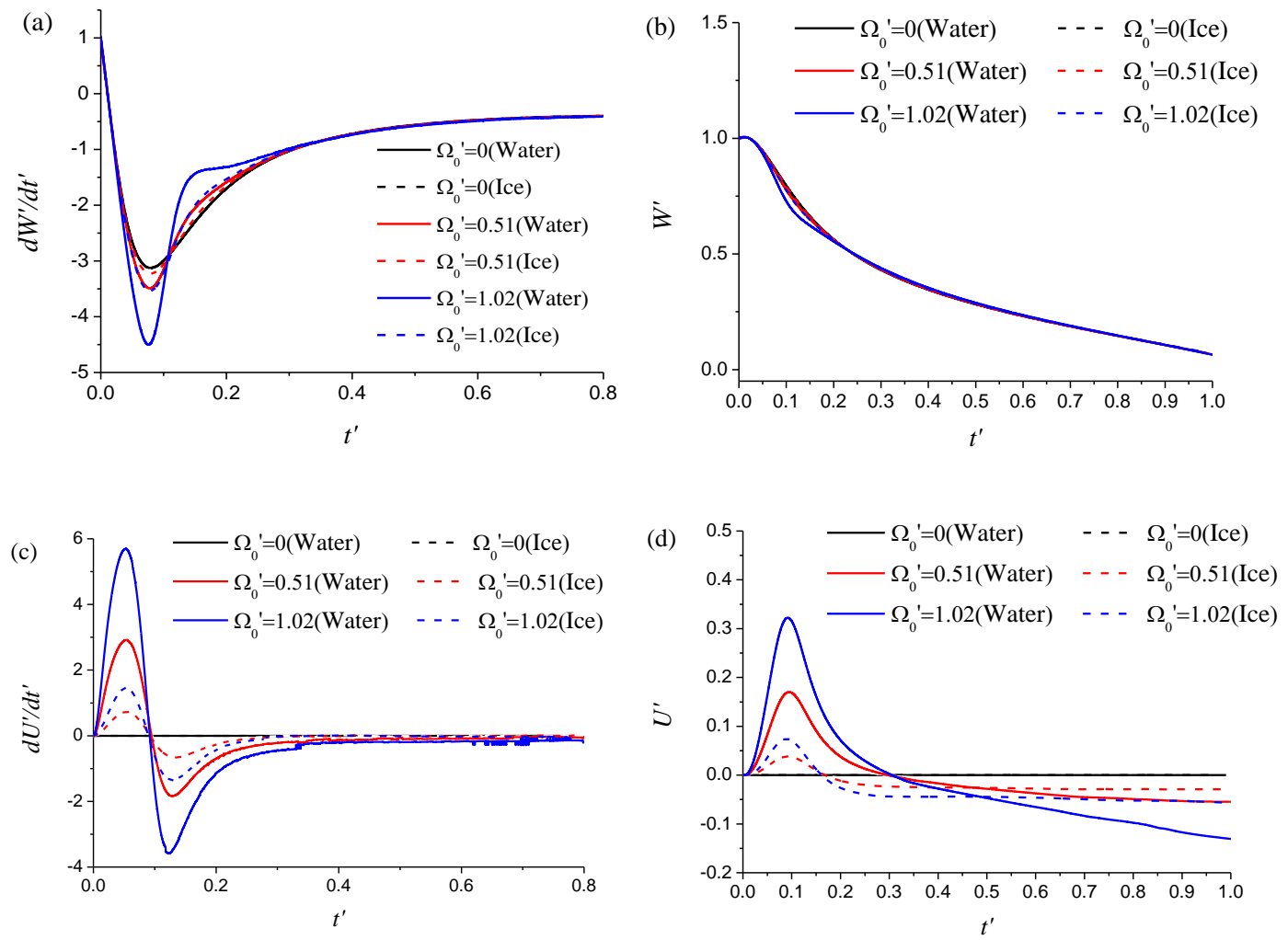

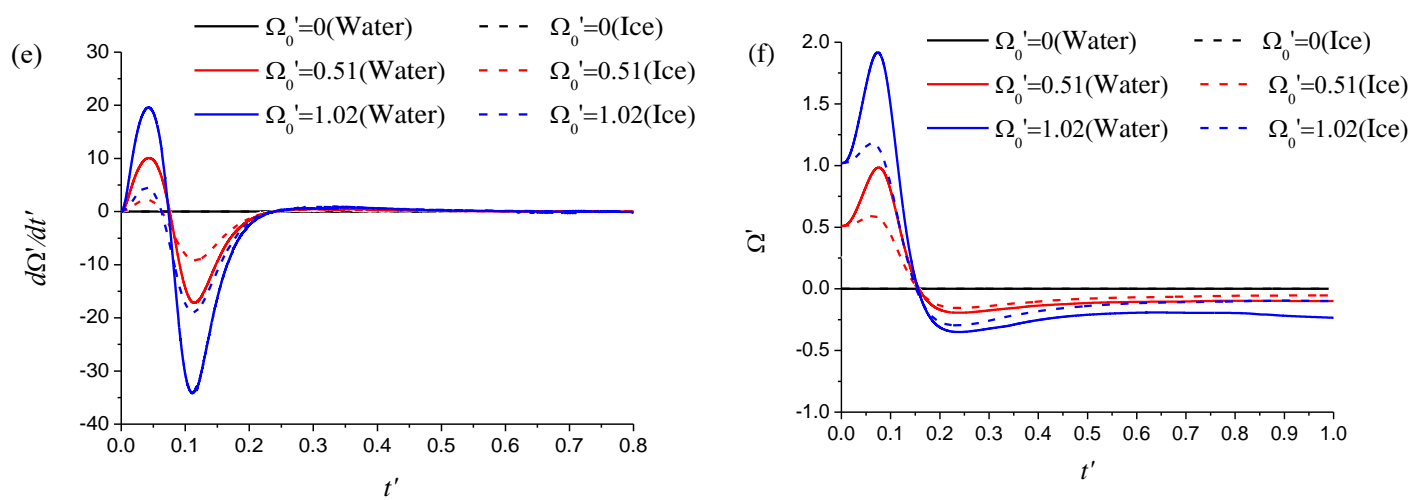

Fig. 8. Water entry of a wedge tank $\left(\gamma_{0}=\pi / 4, \theta_{0}=0, H_{1}^{\prime}=0.196\right)$ at different initial angular velocity $\left(U_{0}^{\prime}=0, W_{0}^{\prime}=1\right)$ with water and ice block respectively, accelerations: (a) vertical, (b) horizontal and (c) rotational; velocities: (d) vertical, (e) horizontal and (f) rotational.

We may also investigate the rationality of the assumption which treats the inner liquid stationary. Fig. 8 shows that in terms of velocity the effect of the inner liquid is mainly on the horizontal and rotational motions, and they are replotted in Fig. 9 with the results of stationary water assumption. For a positive $\Omega_{0}^{\prime}$, the wedge rotates in the anticlockwise direction. The pressure on the left hand side of the wedge is larger, and therefore it pushes the body in the $x$ direction. The stationary liquid seems to be 'lighter' than the frozen ice block, and the horizontal velocity increases faster. When the liquid is in motion, its motion effect makes velocity increase even faster. The result strongly suggests that the movement of the inner liquid has major effect on the body motion and it has to be accounted for properly. Similar pattern can be observed in rotational motion. When the wedge rotates in the anticlockwise direction, the inner liquid will move to the left hand. This will further increase the rotational motion as can be observed in Fig. 9(b).
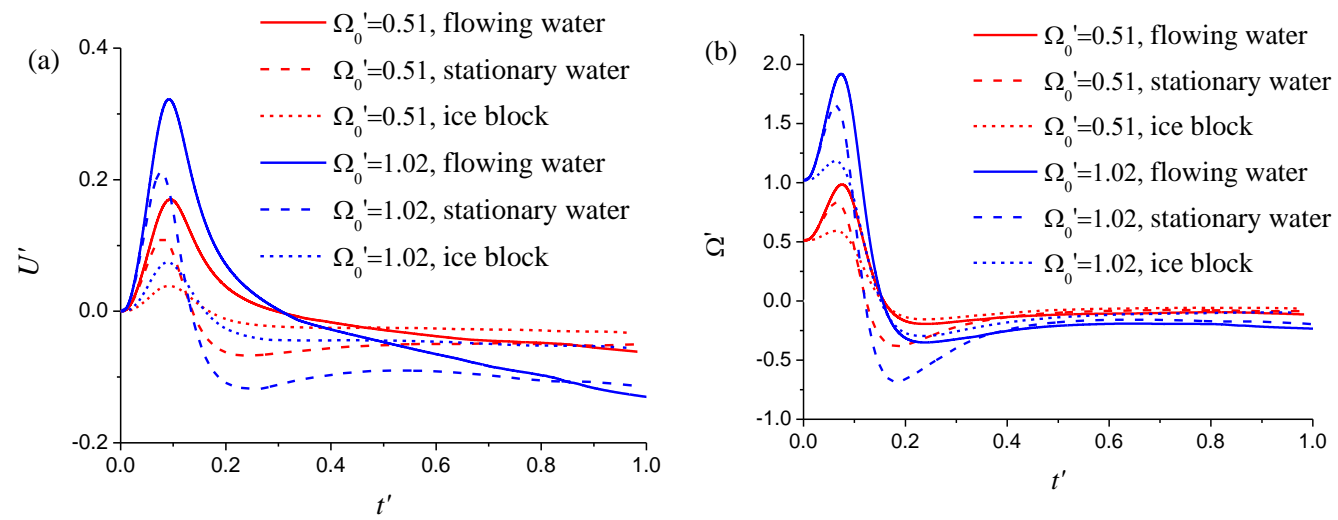

Fig. 9. Water entry as in Fig. 8: (a) The horizontal velocity and (b) rotational velocity

Fig. 10 shows the pressure distribution on the outer surface of the wedge tank for the cases in Fig. 8. They are given when the vertical distance the wedge has travelled reached at $s^{\prime}=0.02,0.04$, $0.08,0.12$ and 0.27 respectively. When $\Omega_{0}^{\prime}=0$, the external pressure distributions on the tanks with water and ice are the same, as in such case the vertical velocity is same, as discussed in Fig. 8. When 
$\Omega_{0}^{\prime} \neq 0$, based on the discussion in Fig. 8 , we can find that the tank containing water has a larger anticlockwise rotational velocity and acceleration. This would make the relative velocity between the wedge and water on the left hand side become larger, and correspondingly the deadrise angle on the left hand side becomes smaller. All these could make the impact pressure on the left hand side become larger. Intuitively, one might expect that on the left hand side, the pressure would be larger when the deadrise angle is smaller. However the pressure for the tank containing ice is larger, which in fact corresponds to larger deadrise angle. Here in addition to the rotation, the body simultaneously moves in the $+x$ direction. The tank with water moves faster than that with ice. This would lead the pressure on left hand side with water to decrease, and become even smaller than that with ice. That indicates that in some cases the movement of the inner liquid may increase the motion of the tank, but it may not increase the impact pressure. As the velocity magnitude of tank decreases at the later stage, the hydrostatic pressure becomes more important, and thus the pressure distributes approximately linearly. Also the rotational angles in the cases with water and ice tend to the same, and the pressures in Fig. 10(e) are therefore similar. Rapid variation can be observed in Fig. 10(e), especially inside the thin layer liquid along the wedge surface. Finer mesh and smaller time step for $\Omega_{0}^{\prime}=0$ have been used to investigate specifically this oscillation, and results are shown in Fig. $10(\mathrm{f}, \mathrm{g})$, which corresponds to $W^{\prime}=0.458$. It can be seen that smaller time step or finer mesh does not affect the oscillation of pressure in the thin layer at the jet root, and this implies that the oscillation is not because of non-convergence.
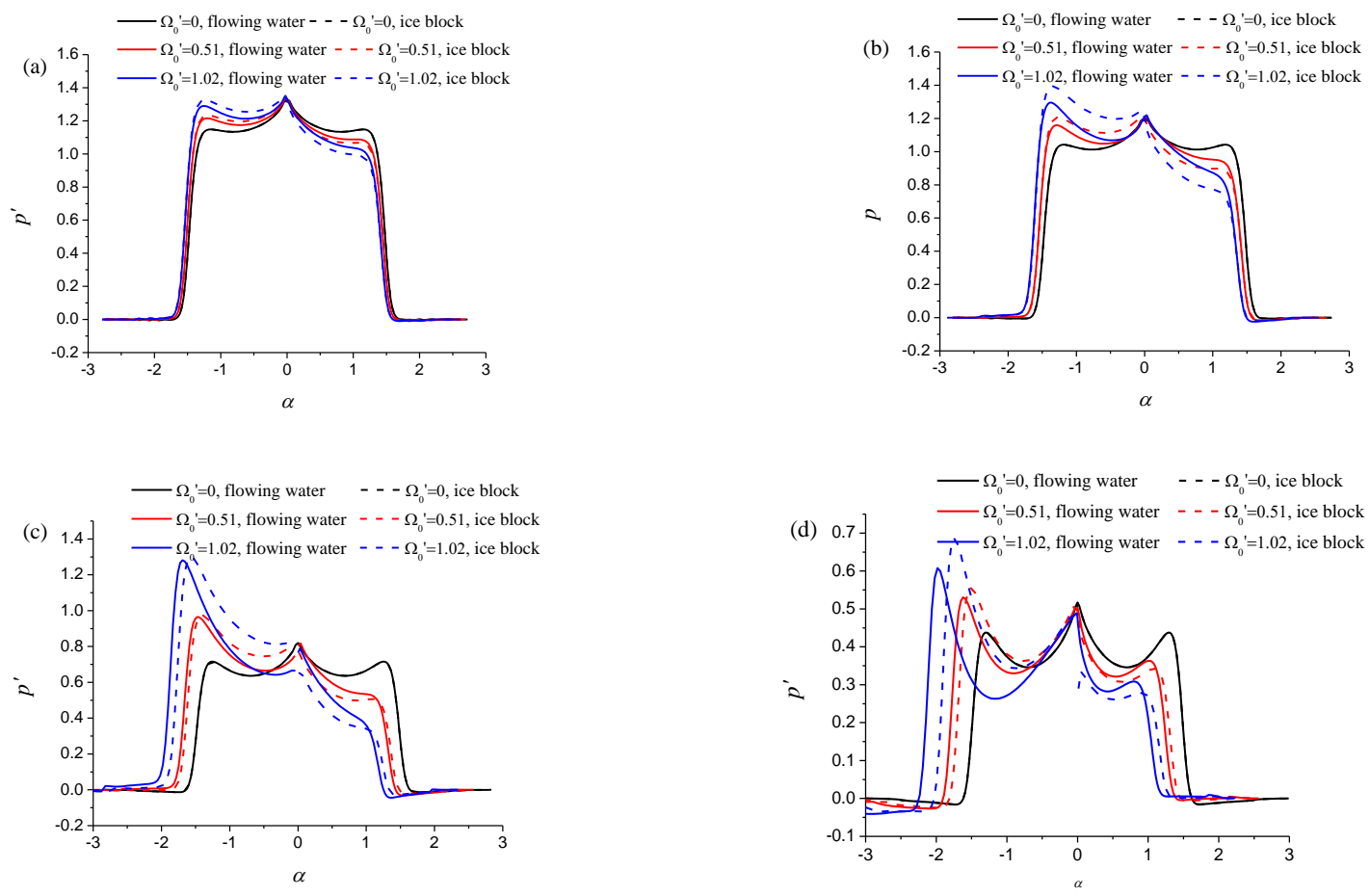

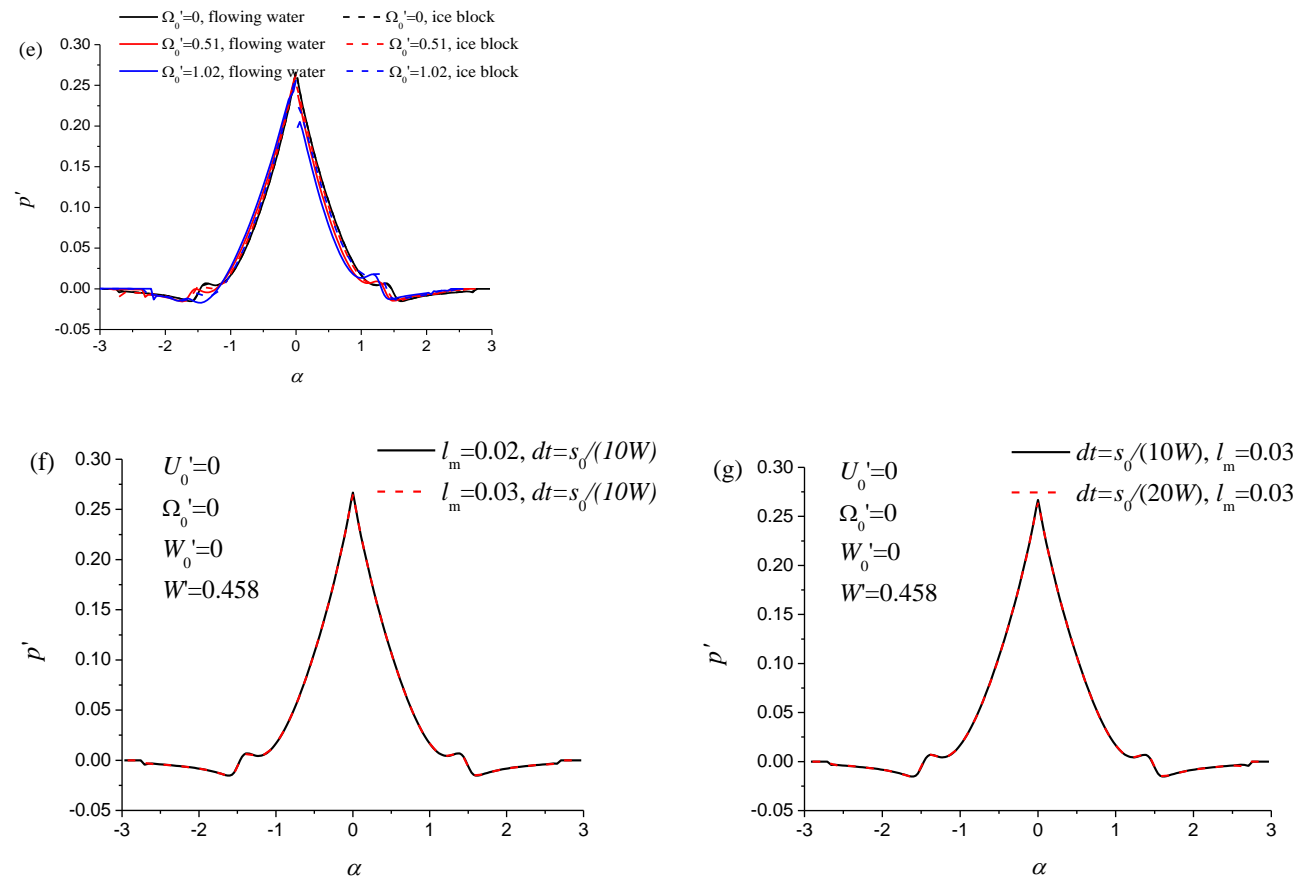

Fig. 10. The outer pressure distribution for the wedge surface at $W_{0}^{\prime}=1$ and $U_{0}^{\prime}=0$ and different initial angular velocities $\Omega_{0}^{\prime}$ : (a) $s^{\prime}=0.02$, (b) $s^{\prime}=0.04$, (c) $s^{\prime}=0.08$, (d) $s^{\prime}=0.12$, (e) $s^{\prime}=0.27$, (f) finer mesh for $\Omega_{0}^{\prime}=0$ in (e), (g) smaller time step for $\Omega_{0}^{\prime}=0$ in (e).

Figs. 11 depicts the free surface profile inside the wedge tank. Free surface deformation becomes significant as the body rotates. In Fig. 11(a), the inner free surface remains approximately stationary relative to the tank. However, in Fig. 11(c), the free surface deformation becomes evident and there is a wave moving from the right hand side to the left. When the tank changes its rotational direction, the leftward motion of the free surface is suddenly blocked. As a result, the free surface moves upwards along the tank wall and a relatively thin layer of the fluid has formed in Fig. 11(d). The leftward motion of free surface can also be used to explain the phenomenon in Fig. 8, which shows the wedge containing water has a larger motion response.
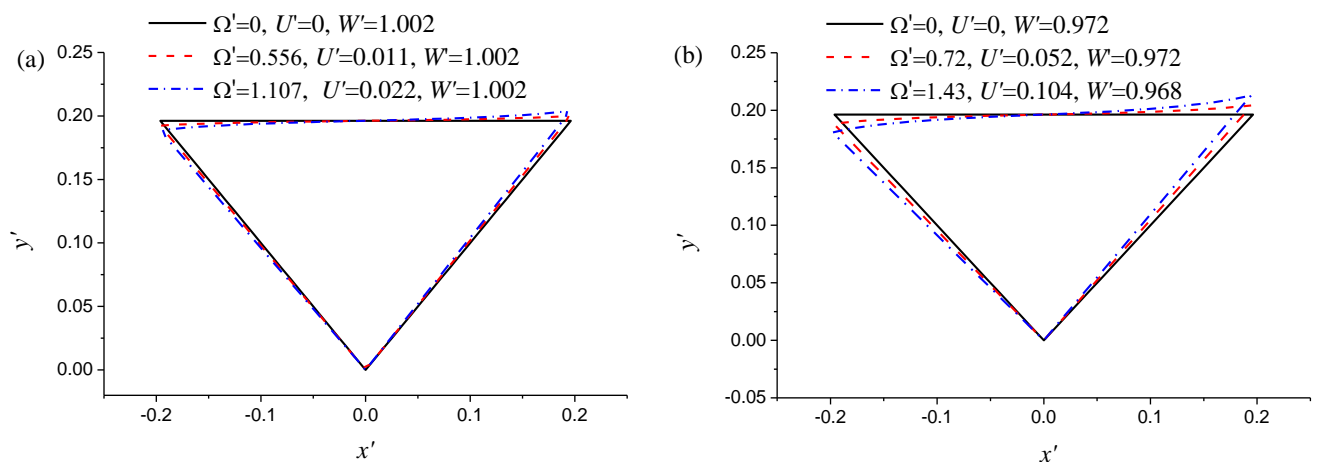

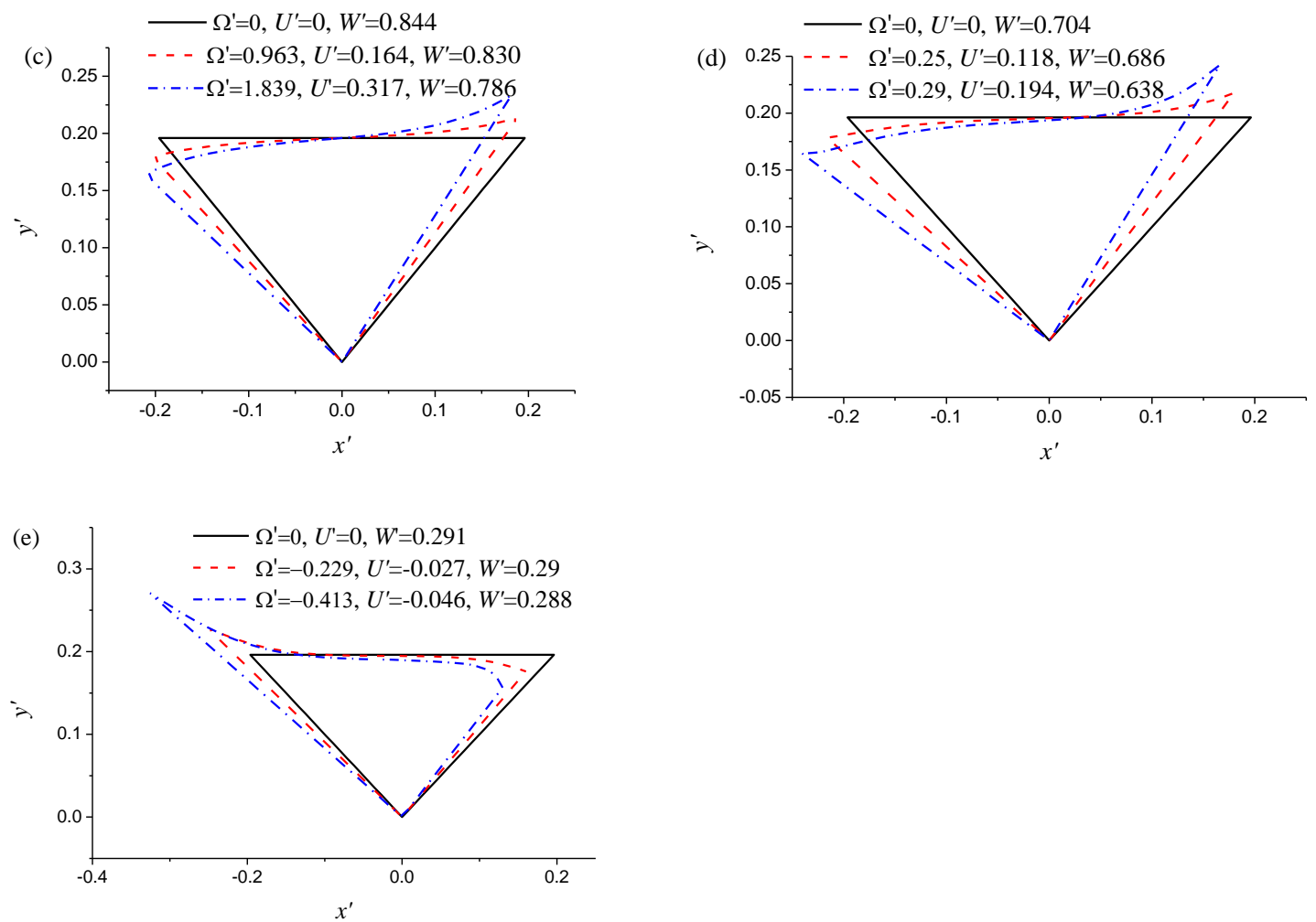

Fig. 11. The inner free surface profile for wedge tank at $W_{0}^{\prime}=1$ and $U_{0}^{\prime}=0$ and different initial angular velocities $\Omega_{0}^{\prime}$ : (a) $s^{\prime}=0.02$, (b) $s^{\prime}=0.04$, (c) $s^{\prime}=0.08$, (d) $s^{\prime}=0.12$, (e) $s^{\prime}=0.27$, (solid line: $\Omega_{0}^{\prime}=0$, dashed line: $\Omega_{0}^{\prime}=0.51$, dash dotted line: $\Omega_{0}^{\prime}=1.02$ ).

Fig. 12 shows the pressure distribution on the inner liquid wedge surface. At $\Omega_{0}^{\prime}=0$, the pressure is linearly distributed along the wedge surface, due to the fact that the internal water is stationary relative to the tank. When $\Omega_{0}^{\prime}>0$, the pressure on the left hand side becomes larger than that at $\Omega_{0}^{\prime}=0$, which is due to the leftward motion of inner water, while the pressure on the right hand side becomes smaller than that at $\Omega_{0}^{\prime}=0$, except in a small area near the water surface, where the mutual interaction between the static water and suddenly moving body at $t^{\prime}=0$ makes the pressure become larger, as can be seen in Fig. 12(a, b). When $t^{\prime}$ is not small, the larger pressure near the water surface disappears, as can be seen in Fig. 12(c $\sim$ e). With the water rising up along the left tank wall, a thin layer of liquid forms. The pressure along the thin layer is close to atmospheric one, as can be seen in Figs. 12(d) and (e). 

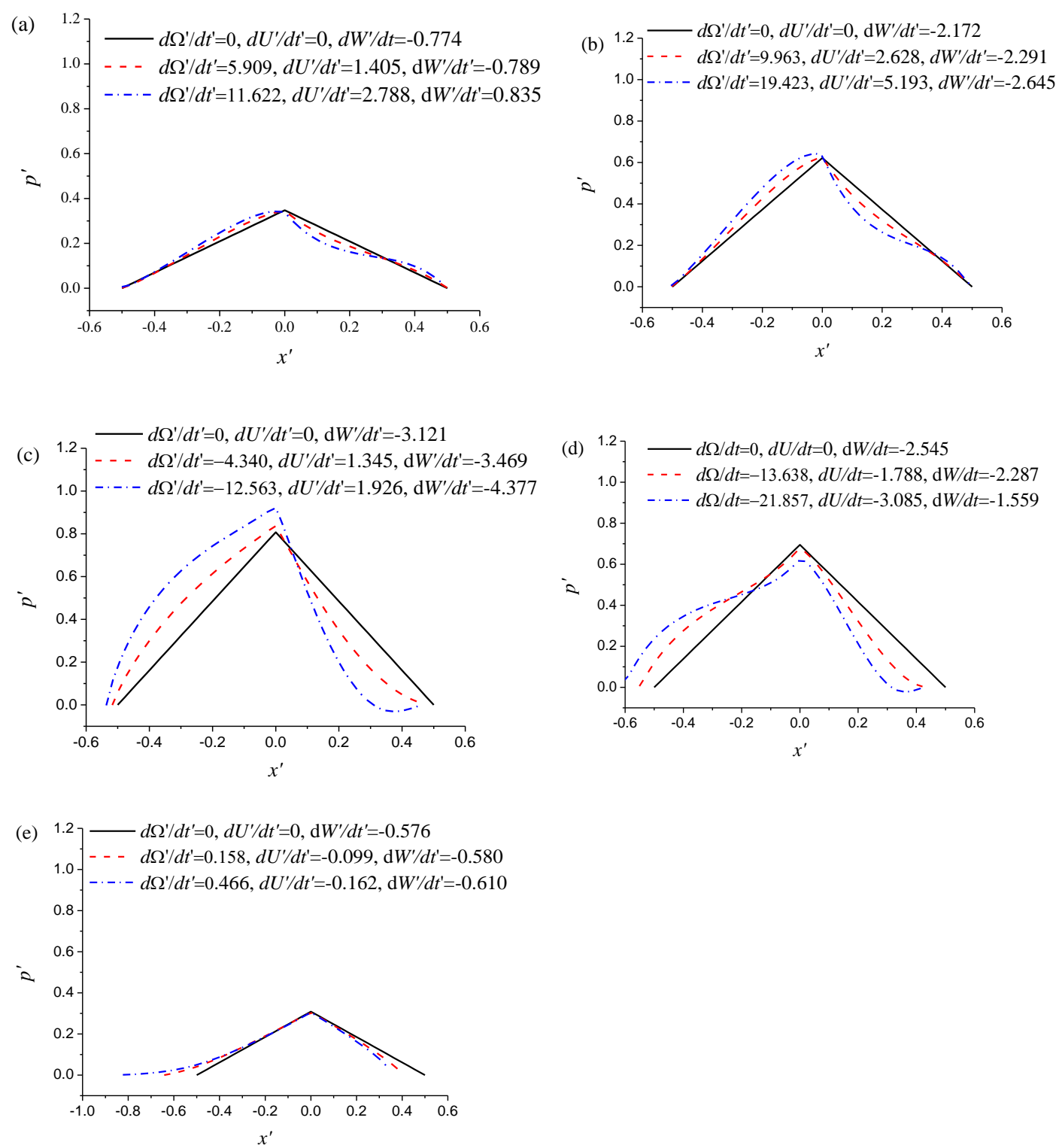

Fig. 12. The pressure distribution along the inner wedge wall at $W_{0}^{\prime}=1$ and $U_{0}^{\prime}=0$ and different initial angular velocities $\Omega_{0}^{\prime}$ : (a) $s^{\prime}=0.02$, (b) $s^{\prime}=0.04$, (c) $s^{\prime}=0.08$, (d) $s^{\prime}=0.12$, (e) $s^{\prime}=0.27$, (solid line: $\Omega_{0}^{\prime}=0$, dashed line: $\Omega_{0}^{\prime}=0.51$, dash dotted line: $\Omega_{0}^{\prime}=1.02$ )

\section{Conclusions}

The problem of water entry of a wedge tank filled with liquid in free fall motion has been solved using the boundary element method, based on velocity potential theory with fully nonlinear boundary conditions. Extensive simulations have been undertaken for a wedge tank with liquids of different densities and depth, as well as frozen ice respectively, from which the following conclusions can be drawn.

(1) There are two time scales corresponding to water entry and sloshing motion inside the tank respectively. The sloshing period is usually much larger than the duration of interest in water entry. This implies that the effect of the liquid is mainly due to its initial motion rather than the full 
oscillatory sloshing motion, especially at a larger $H_{1}^{\prime}$.

(2) The oscillation of vertical velocity is mainly due to the variation of impact force rather than the oscillation of internal water. In contrast to the case with ice, the motion of the inner liquid can make the tank far more responsive to the impact force, especially in horizontal and rotational motions. An exception is that when there is vertical motion only, in which the free surface will remain stationary relative to the tank, and the motion with the liquid is the same as that with ice. In general, while the earlier results may be different, as time increases, the results of both velocities and accelerations with liquid tend to those with ice.

(3) At different $\rho_{1}^{\prime}$, the trough of vertical velocity and the subsequent peak both occur almost at the same time. Similar patterns can be seen in horizontal and rotational velocities. This is because the natural periods at different densities with the same fluid depth are the same and the internal liquid motion follows a similar time scale. As $\rho_{1}^{\prime}$ increases, the magnitude of maximal vertical velocity becomes larger because of larger weight, while the magnitudes of maximal horizontal velocity and rotational velocity become smaller due to larger mass.

(4) The impact pressure depends on the deadrise angle and relative velocity between wedge surface and fluid, as in general water entry problems. The relative velocity is affected by motions in three degrees of freedom. The internal liquid has different effect on each of these three modes, which makes its overall effect on the impact pressure more complex. It is possible at smaller deadrise angle the impact pressure may be smaller.

(5) As the duration of impact is short, the motion of the inner liquid is mainly towards one side. It is expected for a longer duration, the oscillatory sloshing motion of the liquid will have a major effect and the velocity of the wedge may reverse its direction. These will be investigated in the future work.

\section{Acknowledgement}

This work is supported by Lloyd's Register Foundation through the joint centre involving University College London, Shanghai Jiaotong University and Harbin Engineering University, to which the authors are most grateful. Lloyd's Register Foundation helps to protect life and property by supporting engineering-related education, public engagement, and the application of research.

This work is also supported by the National Natural Science Foundation of China (Grant No. 51879123, 51809123) and the Key University Science Research Project of Jiangsu (18KJA130001).

\section{References}

[1] S.D. Howison, J.R. Ockendon, S.K. Wilson, Incompressible water-entry problems at small deadrise angles, J. Fluid Mech. 222 (1991) 215-230.

[2] A. Korobkin, R. Guéret, R. Malenica, Hydroelastic coupling of beam finite element model with Wagner theory of water impact, J. Fluids Struct. 22 (2006) 493-504.

[3] A.A. Korobkin, Second-order Wagner theory of wave impact, J. Eng. Math. 58 (2007) 121139.

[4] Z.N. Dobrovol'skaya, On some problems of similarity flow of fluid with a free surface, J. Fluid Mech. 36 (1969) 805-829.

[5] R. Zhao, O.M. Faltinsen, Water entry of two-dimensional bodies, J. Fluid Mech. 246 (1993) 593-612.

[6] Y.A. Semenov, A. Iafrati, On the nonlinear water entry problem of asymmetric wedges, J. Fluid Mech. 547 (2006) 231-256.

[7] G.D. Xu, W.Y. Duan, G.X. Wu, Numerical simulation of oblique water entry of asymmetrical 
wedge, Ocean Eng. 35 (2008) 1597-1603.

[8] G.X. Wu, Numerical simulation of water entry of twin wedges, J. Fluids Struct. 22 (2006) 99108.

[9] G.X. Wu, H. Sun, Y.S. He, Numerical simulation and experimental study of water entry of a wedge in free fall motion, J. Fluids Struct. 19 (2004) 277-289.

[10] G.D. Xu, W.Y. Duan, G.X. Wu, Simulation of water entry of a wedge through free fall in three degrees of freedom, Proc. R. Soc. A 466 (2010) 2219-2239.

[11] S.Y. Sun, S.L. Sun, G.X Wu, Oblique water entry of a wedge into waves with gravity effect, J. Fluids Struct. 52 (2015), 49-64.

[12] S.Y. Sun, G.X. Wu, G. Xu, Breaking wave impact on a floating body with air bubble effect, J. Fluids Struct. 82 (2018) 16-34.

[13] C.M. Bao, G.X. Wu, G.D. Xu, Simulation of water entry of a two-dimension finite wedge with flow detachment, J. Fluids Struct. 65 (2016) 44-59.

[14] C.M. Bao, G.X. Wu, G. Xu, Water entry of a finite width wedge near a floating body, Appl. Ocean Res. 84 (2019) 12-31.

[15] S.Y Sun, G.X. Wu, G. Xu, Free fall motion of a floating body: bubble formation and its effect, Eur. J. Mech. B Fluids 76 (2019) 178-189

[16] J. Wang, O.M. Faltinsen, Numerical investigation of air cavity formation during the high-speed vertical water entry of wedges, J. Offshore Mech. Arct. Eng. 135 (2013) 011101.

[17] J. Wang, O.M. Faltinsen, Improved numerical solution of Dobrovol'skaya's boundary integral equations on similarity flow for uniform symmetrical entry of wedges, Appl. Ocean Res. 66 (2017) 23-31.

[18] M. Barjasteh, H. Zeraatgar, M.J. Javaherian, An experimental study on water entry of asymmetric wedges, Appl. Ocean Res. 58 (2016) 292-304.

[19] O.M. Faltinsen, A.N. Timokha, Sloshing, Cambridge University Press, 2009.

[20] O.M. Faltinsen, A.N. Timokha, A multimodal method for liquid sloshing in a two-dimensional circular tank, J. Fluid Mech. 665 (2010) 457-479.

[21] O.M. Faltinsen, A.N. Timokha, Analytically approximate natural sloshing modes for a spherical tank shape, J. Fluid Mech. 703 (2012) 391-401.

[22] B. Molin, F. Remy, Experimental and numerical study of the sloshing motion in a rectangular tank with a perforated screen, J. Fluids Struct. 43 (2013) 463-480.

[23] G. Bulian, E. Botia-Vera, A. Souto-Iglesias, Experimental sloshing pressure impacts in ensemble domain: Transient and stationary statistical characteristics, Phys. Fluids 26 (2014) 032102 .

[24] B. Bouscasse, M. Antuono, A. Colagrossi, and C. Lugni, Numerical and experimental investigation of nonlinear shallow water sloshing, Int. J. Nonlinear Sci. Numer. Simul. 14 (2013) 123.

[25] M. Barjasteh, H. Zeraatgar, M.J. Javaherian, An experimental study on water entry of asymmetric wedges, Appl. Ocean Res. 58 (2016) 292-304.

[26] Y.A. Semenov, G.X. Wu, Water entry of a wedge with rolled-up vortex sheet, J. Fluid Mech. 835 (2018) 512-539.

[27] G.X. Wu, R. Eatock Taylor, The coupled finite element and boundary element analysis of nonlinear interactions between waves and bodies, Ocean Eng. 30 (2003) 387-400.

[28] C.H. Lu, Y.S. He, G.X. Wu, Coupled analysis of nonlinear interaction between fluid and 
structure during impact, J. Fluids Struct. 14 (2000) 127-146.

[29] G.X. Wu, Hydrodynamic force on a rigid body during impact with liquid, J. Fluids Struct. 12 (1998) 549-559.

[30] Lamb, Hydrodynamics, 6th Edition, Cambridge University Press, Cambridge, 1932. 